\title{
METHODS AND INSTRUMENTATION FOR BUNCH SHAPE MEASUREMENTS
}

\author{
A.V.Feschenko, Institute For Nuclear Research, Moscow 117312, Russia
}

\begin{abstract}
Bunch shape is one of the most important and interesting characteristics of a beam in ion linear accelerators. Recently detectors of bunch shape with a transverse scanning of low energy secondary electrons found application in several accelerators. The principle of operation of the detectors is based on a coherent transformation of a longitudinal structure of a beam under study into a transverse distribution of a secondary electron beam through $\mathrm{rf}$ scanning. There are three modifications of the detectors: Bunch Shape Monitor, Bunch Length (Shape) and Velocity Detector and Three Dimensional Bunch Shape Monitor. The principle of operation of the detectors as well as the peculiarities of the structure and the design of the detectors are described. To demonstrate the possibilities of the detectors the results of measurements with different detectors made at different accelerators are presented.
\end{abstract}

\section{INTRODUCTION}

In this report both a longitudinal charge distribution in bunches and a three-dimensional charge distribution are implied by a term of a bunch shape. The following requirements can be settled to the detectors of bunch shape: high resolution in phase, small dimensions along the beam line, small beam distortion, wide band of measurements in beam intensity and sufficient band in phase, small power consumption. The measurements should be direct and mathematical reconstruction techniques should be avoided. The resolution of $1^{\circ}$ corresponds to the equivalent bandwidth $\Delta F=180 \mathrm{f} / \Delta \varphi$, which for example for $f=200 \mathrm{MHz}$ equals $36 \mathrm{GHz}$. Fast beam monitors interacting with an electromagnetic field of the bunch can not be used for observation of the bunch shape. The difficulties can be overcome if information about the bunch shape is transmitted through some other type of radiation. Different types are possible: Cherenkov radiation [1], detached electrons in case of $\mathrm{H}^{-}$, both high energy ( $\delta$-electrons) [2] and low energy secondary electrons. Photo-detachment by a laser beam is also possible [3]. Despite the variety of the possibilities the only low energy secondary electrons have found a practical use.

The distinctive feature of these electrons is a weak dependence of their properties both on the type of primary particles and on the energy of the analysed beam. Due to this feature the detectors can be used for almost any ion beam. Among the characteristics of low energy secondary emission, influencing the parameters of the bunch shape detector, one can mark out initial energy and angular distributions as well as time dispersion or delay of the emission. Time dispersion establishes a fundamental limitation of a resolution of the detector. The value of time dispersion for metals is estimated theoretically to be $10^{-14} \mathrm{~s}$, the theoretical lower limit being $10^{-15} \mathrm{~s}$ [4]. The experimental attempts to measure the time dispersion give the upper limit within the range from several ps to several hundred ps depending on the resolution of the measuring equipment [4].

Operation of all the bunch shape monitors with low energy secondary electrons is based on coherent transformation of a time structure of the analysed beam into a spatial distribution of secondary electrons through rf modulation. The first real detector has been built by R.Witkover [5]. In this detector the electrons emitted from a thin strip target are accelerated by electrostatic field and simultaneously modulated in energy by rf electric field. Further analysis in a magnetic field enables to spatially separate the electrons with different energies, each point of the spatial distribution corresponding to a particular point of the longitudinal distribution of the analysed beam. The detector [6] uses the same principle except for separation in space of the processes of electrostatic acceleration and $\mathrm{rf}$ modulation. Both the above detectors use $\mathrm{rf}$ modulation in energy or in other words a longitudinal modulation. Another possibility is using of a transverse scanning. The electrons can be modulated in the transverse direction and deflected depending on their phase. Spatial separation can be obtained after a drift space. Transverse modulation was successfully used in the fifties to measure the upper limit of time dispersion of the secondary emission [7]. In the first proposal of a bunch shape detector with transverse modulation of low energy secondary electrons, made in the early sixties [8], a circular scan with the help of two rf deflectors is foreseen. The circular scan provides a band of measurements equal to a full period of the rf deflecting field. However the bunches are normally much shorter than the period and instead of a circular scan one can use a linear one thus essentially simplifying the detector.

\section{BUNCH SHAPE MONITOR WITH TRANSVERSE SCANNING}

The first real detector of a longitudinal distribution with transverse scanning of low energy secondary electrons, developed and fabricated in INR, has been described in [9]. This detector was used during the commissioning of the INR linac [10]. Now it is installed 
in the $100 \mathrm{MeV}$ transition region. The disadvantage of this detector is unstable operation of its deflector due to multipactor discharge. The solution of the problem has been found by combination of the rf deflector with the electrostatic lens [11]. This solution is used in all the subsequent bunch shape monitors. The schematic diagram of the detector is shown in fig. 1.

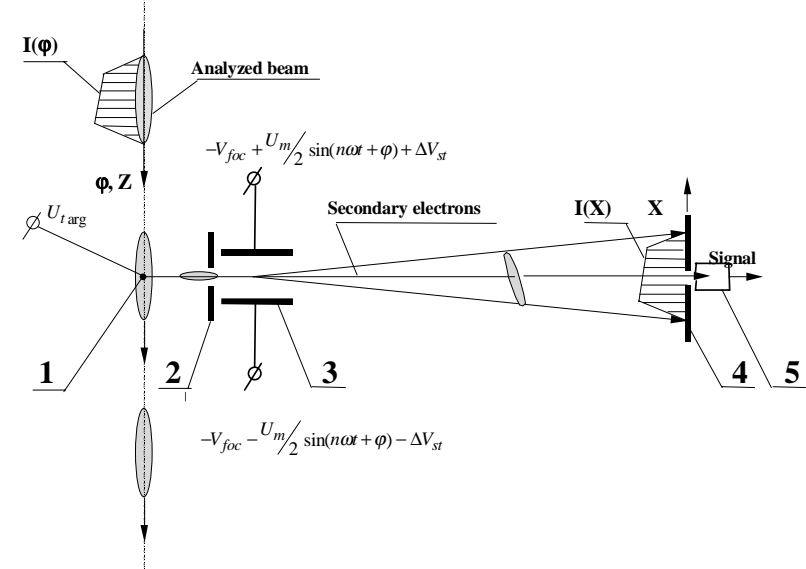

Figure 1: General configuration of Bunch Shape Monitor (1-wire target, 2-input collimator, 3-deflector, 4-output collimator, 5-electron collector).

Phase resolution of the detector is defined as $\Delta \varphi=\Delta X / X_{\max }$, where $\Delta X$ is a beam size for a monophase bunch, and $X_{\max }$ is a maximum displacement of the electrons. To find the value of $\Delta X$ a semiexperimental approach is used: the size of the beam with the turned off deflecting field is measured experimentally and the influence of the deflecting field is found by simulations. The value of $\Delta \varphi$ depends on many parameters but normally a value better than $1^{\circ}$ for the frequencies of hundreds $\mathrm{MHz}$ can be obtained.

\section{MODIFICATIONS OF THE DETECTOR}

Besides the Bunch Shape Monitor (BSM) there are two modifications of the detector: Bunch Length and Velocity Detector (BLVD) and Three Dimensional Bunch Shape Monitor (3D-BSM).

BLVD is a BSM, which can be mechanically translated along the beam line [12]. In this detector a time of flight method of energy measurements is implemented. The translation results in a horizontal shift of the observed distribution. Measuring the value of the translation and the value of the shift one can find an average velocity of the beam. To decrease systematic errors of measurements the distance of translation must be equal to $\frac{\beta \lambda_{d}}{2}$ and it must be carefully calibrated preliminary. Here $\lambda_{d}$ is a wavelength of the deflecting field. Bunch shape must be measured before and after the translation of the detector for the phase ranges shifted by $180^{\circ}$. Before the measurement of a the bunch shape the electron beam must be carefully steered to fit exactly into slit 4 with the deflecting field turned off for each position of the detector. With the above procedure the systematic error can be decreased to $\pm 0.1 \%$. The total error is typically within $\pm(0.3 \div 0.4) \%$.

3D-BSM is aimed to measure a three dimensional distribution of charge in bunches [13,14]. Due to high strength and concentration of electric field near the wire the electrons move almost perpendicular to its axis. Fig. 2 shows distribution of co-ordinates after electrostatic acceleration for the electrons escaped from the target at a fixed co-ordinate as well as FWHM of the distribution versus target potential for different target to collimator distances.
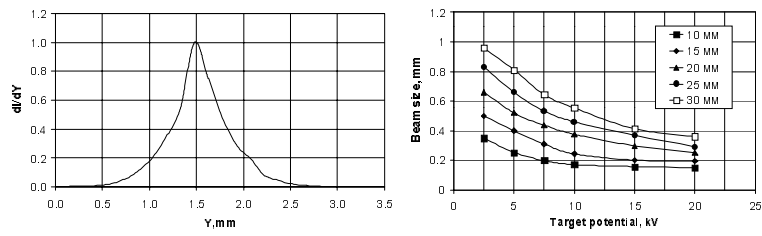

Figure 2: Broadening of secondary electron beam.

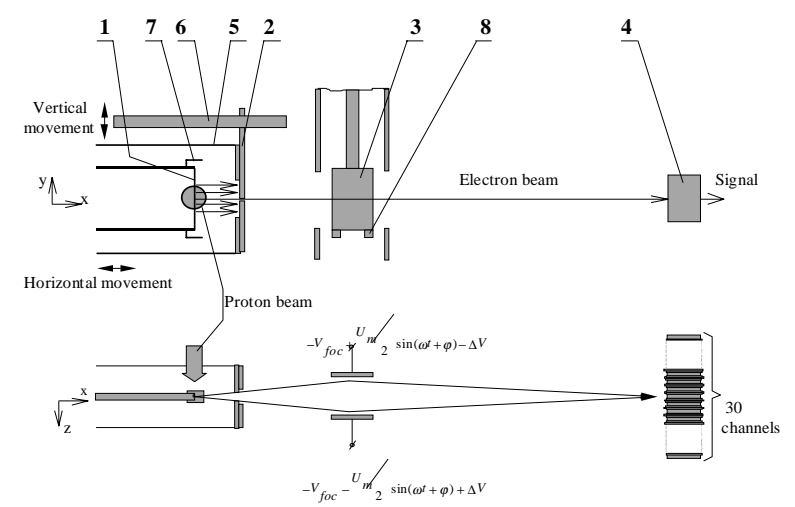

Figure 3: General configuration of 3D-BSM (1-target, 2plate of horizontal slit, 3-deflector plates, 4-multi-channel electron collector, 5-electrostatic screen, 6-guide, 7,8-field correcting elements).

Principle of operation of the 3D-BSM is explained in fig.3. Longitudinal distribution of the electrons is measured with multi-channel collector 4 . Moving the target along with the elements 5 and 2 horizontally and slit 2 vertically and measuring longitudinal distribution for each transverse point one can get a three-dimensional distribution. If the signal from the multy-channel collector is digitised within the beam pulse the result of the measurement is a four-dimensional function $J(x, y, \varphi, t)$.

Up to now seventeen detectors with transverse scanning of low energy secondary electrons have been built in different laboratories.

\section{REMARKS ON DESIGN OF THE DETECTORS}

Though the principle of operation of all the detectors is similar the variety of mechanical and electrical requirements as well as the variety of beam parameters result in essentially different implementation of the 
detectors. Figures $4 \div 6$ give an idea about possible implementations of the detectors.

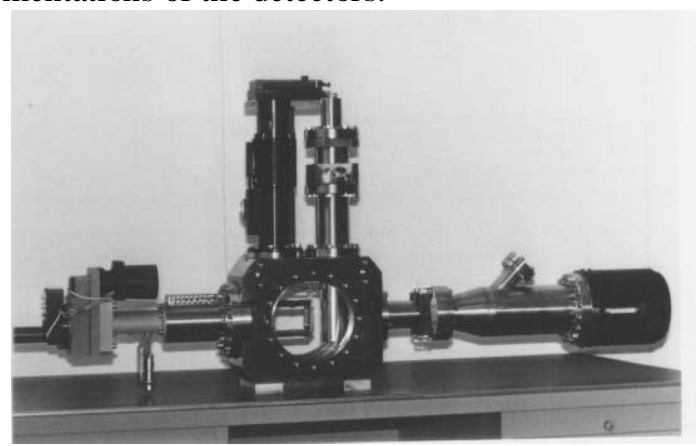

Figure 4: 3D-BSM for CERN Linac-2.

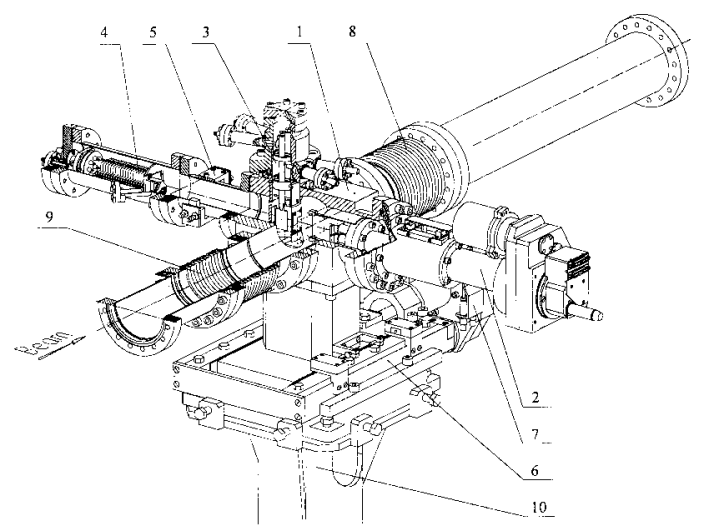

Figure 5: 3D view of the BLVD for DESY Linac-3.

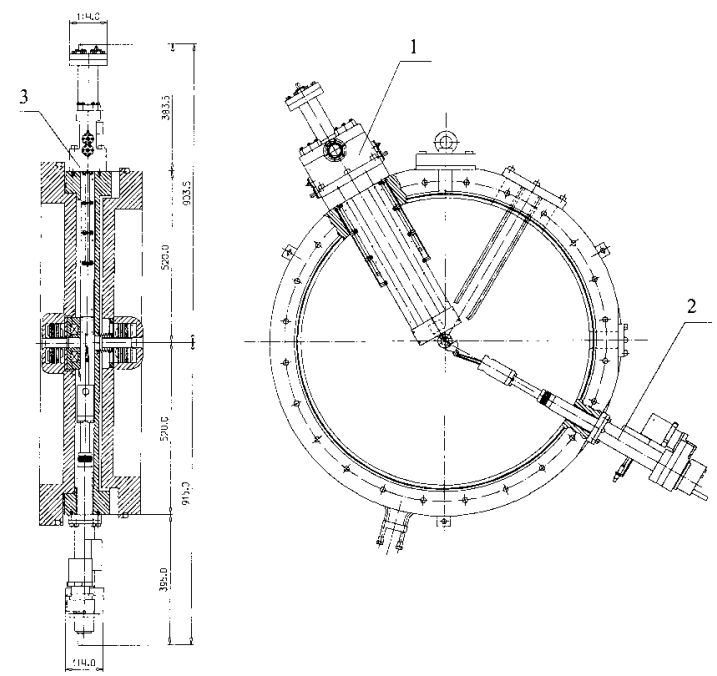

Figure 6: BSM for CERN Linac-2 and DESY Linac-3.

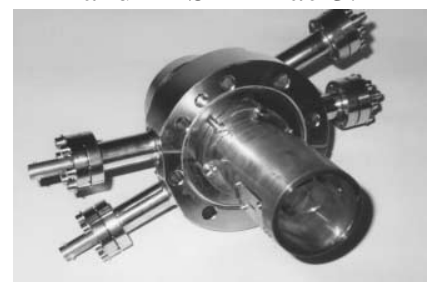

Figure 7: Rf deflector of the INR BLVD.

The detector in fig. 6 is designed to fit into the spare ports in the inter-tank sections.
The main element of any bunch shape monitor is $\mathrm{rf}$ deflector. The design of the deflector must provide a possibility to supply a HV focusing potential to the plates and an additional potential difference between the plates to steer the electrons. Usually the cavities based on coaxial or parallel wire lines are used. Fig.7 shows the picture of the deflector for the INR BLVD ( $f=991 \mathrm{MHz})$. Typical rf power consumption is about $10 \mathrm{~W}$.

\section{SOME EXPERIMENTAL RESULTS}

In this section some results of measurements made with different detectors at different accelerators are presented.

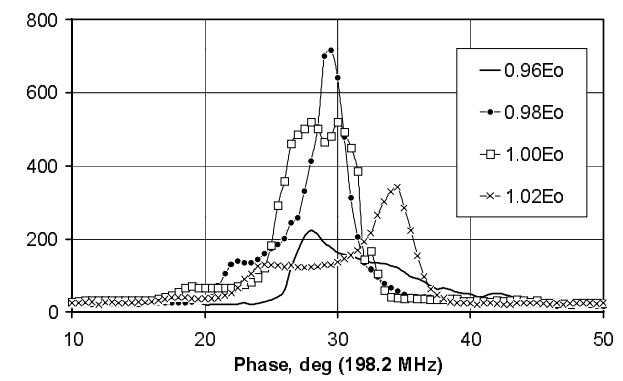

Figure 8: Bunch shapes at the exit of the first tank of INR linac for different accelerating fields.

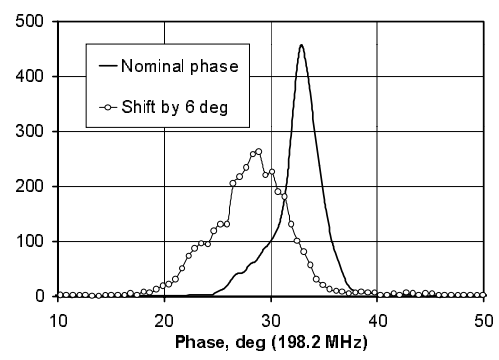

Figure 9: Bunch shapes at the transition region (100 $\mathrm{MeV}$ ) of INR linac.

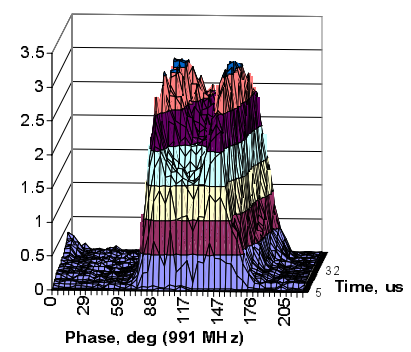

Figure 10: Example of incorrect tuning of accelerator.

Fig.8 shows the results of one of the earliest measurements made at the exit of the first cavity (20 $\mathrm{MeV}$ ) of the INR linac with the first BSM with transverse scanning. These results have been used for precise setting of the amplitude of accelerating field. This BSM is in operation for thirteen years. Now it is used for longitudinal matching of the beam at the transition region from the low energy part to the high energy part of the accelerator. Routinely it is used to check the bunch shape, which is a good indicator of proper or incorrect tuning of the upstream part of the accelerator. Fig. 9 demonstrates 
the effect of changing of the shape due to shift of the phase in one of the accelerating tanks.

INR linac is also equipped with the BLVD installed in the intermediate energy extraction region $(160 \mathrm{MeV})$. An example of experimental data obtained with this detector is presented in fig. 10. Two maximums indicate incorrect tuning of the accelerator.

Four BSMs have been built for the SSC linac [15]. Unfortunately only the first, a test monitor, was in operation at the RFQ test stand [16]. Bunch shapes measured for nominal and small beam current $(<1 \mathrm{~mA})$ are shown in fig. 11. In case of small current a fine structure is observed.
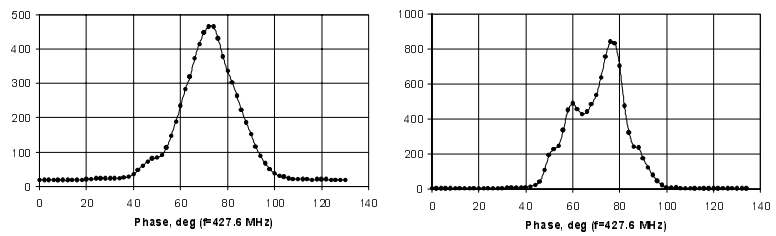

Figure 11: Bunch shapes at the exit of the RFQ of the SSC linac.

Fig.12 demonstrates the result of measurements of a beam energy at the exit of the JHP RFQ [17]. In this detector to avoid overheating of the target the water cooled movable collimator is foreseen in front of the target.

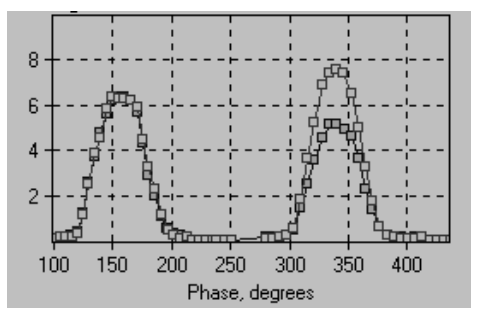

Figure 12: Energy measurement at the exit of the JHP RFQ ( $f=432 \mathrm{MHz})$.

The measurements in CERN Linac-2 a made with the beam pulse current up to $170 \mathrm{~mA}$ [18]. Due to overheating of the target even by a single beam pulse the measurements are made for a position of the target at the edge of the beam. The results of measurements are demonstrated in fig. 13 .
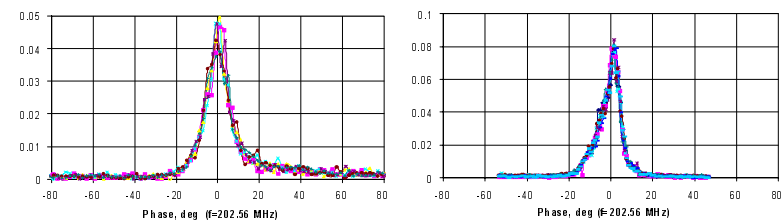

Figure 13: Multiple measurements of bunch shapes in CERN Linac-2 at $10 \mathrm{MeV}$ and $30 \mathrm{MeV}$.

The result of measurement of a three-dimensional distribution is a four dimensional array $J\left(x_{i}, y_{j}, \varphi_{k}, t_{l}\right)$. Processing the experimental data one can get different functions starting from a simple beam pulse (which of course can be observed by conventional means) to quite exotic characteristics as, for example, a behaviour in time of a longitudinally-transversal distribution. Fig.14 demonstrates some of the functions that can be obtained after processing the of the results of a single measurement.

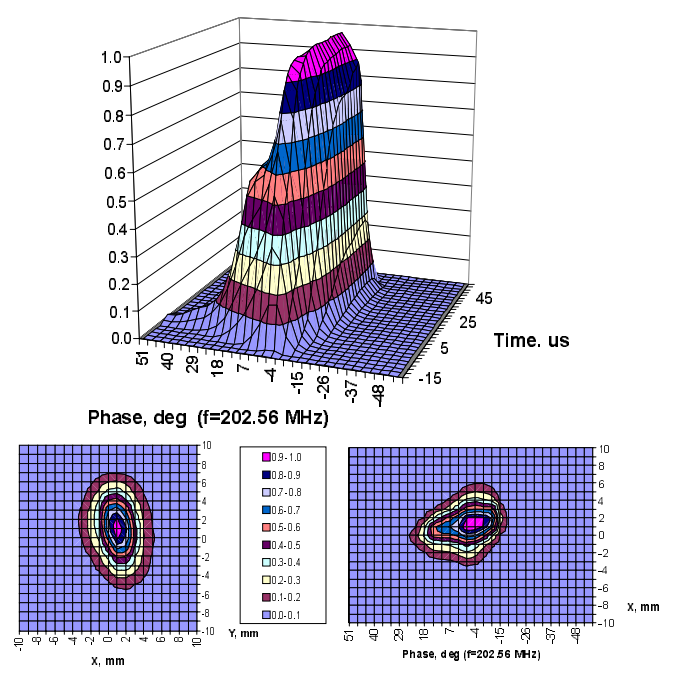

Figure 14: Behaviour of bunch shape in time, beam cross-section and longitudinally-transversal distribution measured at the exit of CERN Linac-2 with the 3D-BSM.

\section{SOME PECULIARITIES OF THE DETECTORS}

In our practical work the target has been destroyed twice and in both cases it has happened for low energies when the range of ions in the target was much smaller than its thickness. The reason can be a non-uniform heating of the target resulting in extra mechanic strength leading to cracks and as a result to deterioration of heat transfer. Fig. 15 shows the temperature distribution across the tungsten wire at the end of the $10^{\text {th }}$ beam pulse after switching the beam on calculated for proton beam with the parameters: $W=2.5 \mathrm{MeV}, I=19 \mathrm{~mA}, T=100 \mu \mathrm{s}$, $F=1 \mathrm{~Hz}, \sigma_{x}=2.0 \mathrm{~mm}, \sigma_{y}=1.5 \mathrm{~mm}$.

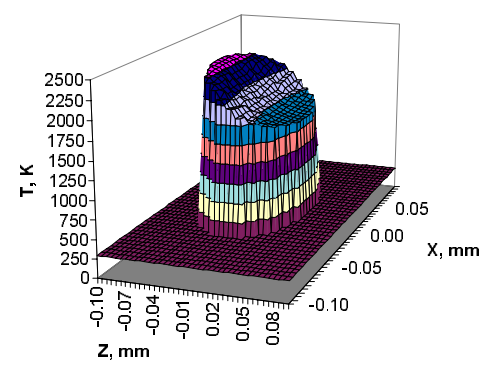

Figure 15: Distribution of temperature across the wire.
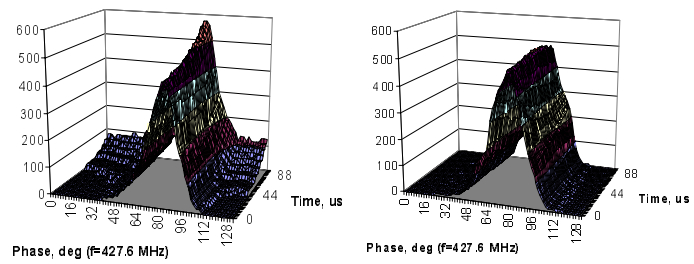

Figure 16: Influence of thermal emission. 
In case of tungsten the temperature of the target is limited by an influence of thermal emission and is estimated to be $1700 \div 1800 \mathrm{~K}$. Fig. 16 shows the results of measurements of bunch shape evolution along the beam pulse for two beam repetition rates, $1 \mathrm{~Hz}$ and $10 \mathrm{~Hz}$. In the second case the thermal emission results in a background increasing it time during the beam pulse. Increasing of the background to the end of the bunch (in fig. 16 from left to right) demonstrates the effect of heating by a single bunch.

In case of $\mathrm{H}^{-}$ions along with the low energy secondary electrons the detached electrons are present. The majority of these electrons are scattered in the target elastically. The indication for this is that with the target potential turned off they are focused with the focusing potential corresponding to their initial energy. Fig.17 shows the curves measured at DESY Linac-3 for different target potentials. The smaller maximum, which can be better seen in logarithmic scale, is due to the detached electrons. Changing of the target potential results in changing of the difference of time of flight of the two groups of electrons from the wire to the deflector and hence in a relative horizontal shift of the two maximums. Elimination of the detached electrons can be done by energy separation downstream of the output collimator $[15,19]$. This method is not applicable for the energies less than several MeV. However at these energies the influence of the detached electrons is not observed $[16,17]$.

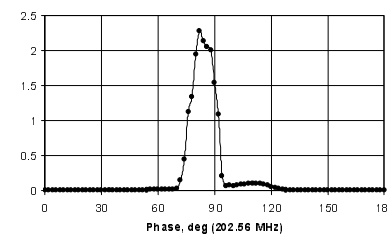

$10 \mathrm{kV}$

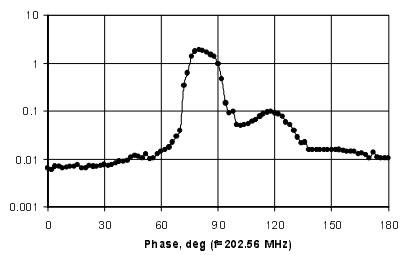

$7 \mathrm{kV}$

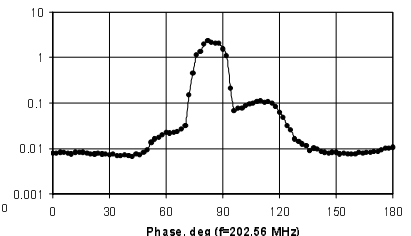

$10 \mathrm{kV}$

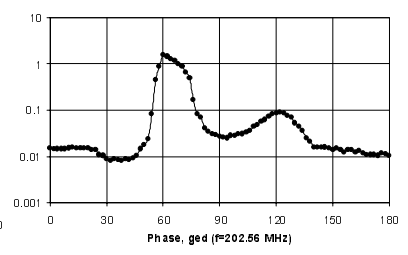

$4 \mathrm{kV}$
Figure 17: Influence of stripped electrons.

Electromagnetic fields of the analysed beam distort the trajectories of the secondary electrons thus resulting in additional errors of measurements [20,21]. The errors are varied along the bunch and are complicated functions of beam current and energy, both longitudinal and transverse parameters of a bunch, target potential and boundary conditions. However our analysis has shown that in practice these effects can be neglected if the ion beam current does not exceed several tens of $\mathrm{mA}$ [21]. In case of extremely high pulse current as it is in the CERN Linac-2 the accuracy degradation up to $2^{\circ}$ is feasible.

\section{SUMMARY}

At present the problem of measurement of bunch shape in ion linac as a whole is solved. The method of measurements based on coherent transformation of longitudinal distribution of ion beam into a spatial one of low energy secondary electrons through rf scanning is applied. There are three modifications of the detector: Bunch Shape Monitor, Bunch Length and Velocity Detector and Three-Dimensional Bunch Shape Monitor. As a rule the phase resolution is better than $1^{\circ}$.

\section{REFERENCES}

[1] Johnson, Kenneth W. USA Patent, 250-639, No.3873839.

[2] A.M.Tron. Collection "Accelerators" No.15., Atomizdat, Moscow, 1976, pp.77-80 (in Russian).

[3] A.S.Artiomov at al. IEEE Part. Acc. Conf., May 6-9, 1991, San Francisco, pp.1573-1575.

[4] Bronstein, I.M., Fraiman, B.S. Secondary Electron Emission, Moscow, Nauka, 1969 (in Russian).

[5] Witkover, R.L. NIM, 1976. V.137, No.2, pp.203-211.

[6] A.M.Tron, A.V.Feschenko. Proc. of the $8^{\text {th }}$ All Union Meeting on Particle Accelerators, v.2, Dubna, 1981, pp. 125-129 (in Russian).

[7] Ernst E.W., H.Von Foerster. JAP, 1955, v.26, No.6, pp.781-782.

[8] I.A.Prudnikov et al. USSR invention license, H05h7/00, No.174281 (in Russian).

[9] A.V.Feschenko, P.N.Ostroumov. Proc. of the 1986 Linear Acc. Conf., Stanford, 1986, pp.323-327.

[10] A.V.Feschenko, P.N.Ostroumov. Proc. of the Workshop on Advanced Beam Instrumentation, KEK, Tsukuba, Japan, April 22-24, 1991, p.236-245.

[11] A.V.Feschenko et al.. Proc. of the EPAC-3 Conf., Berlin, 24-28 March, 1992, v. 2, pp. 1073-1075.

[12] Ostroumov, P.N. INR Preprint-812/92, May 1993 (in Russian).

[13] S.K.Esin, A.V.Feschenko. Proc of the $14^{\text {th }}$ Workshop on Part. Acc., Protvino, October 25-27, 1994, v.2, pp.17-20 (in Russian).

[14] Yu.V.Bylinsky eat al. Proc. of the EPAC-94, London, June 27-July 1, 1994, v. 1, pp.1702-1704.

[15] S.K.Esin et al. Proc of the $14^{\text {th }}$ Workshop on Part. Acc., Protvino, October 25-27, 1994, v.2, pp.21-26 (in Russian).

[16] J.W.Hurd et al. Proc. of the 1993 PAC, Washington, May 1993, pp. 2426-2428.

[17] A.V.Feschenko et al. Proc. of the XIX Int. Linear Acc. Conf., Chicago, IL, August 1998, p.905-907.

[18] C.Hill et al. Proc. of the Int. Linac. Conf. Linac2000, Monterey, Ca, August 21-25, , V. 1, p.590-592.

[19] A.V.Feschenko et al. Proc. of the 1992 Linear Acc. Conf., August 24-28, Ottawa, v. 2, pp. 662-664.

[20] A.Tron, I.Merinov. Proc. of the 1997 PAC, Vancouver, 1997, V.2, pp.2247-2049.

[21] A.V.Feschenko, V.A.Moiseev. Proc. of the Int. Linac. Conf. Linac-2000, Monterey, Ca, August 2125, V.1, p.178-180. 